\title{
The Application of Metrics to Industrial Prototyping Processes: An Empirical Study
}

\author{
Keith Phalp ${ }^{1}$ and Steve Counsell ${ }^{2}$ \\ 1 Empirical Software Engineering Group, Bournemouth University, Bournemouth, \\ UK. email:kphalp@bournemouth.ac.uk \\ 2 Birkbeck College, University of London, Malet Street, London, UK. \\ email:steve@dcs.bbk. ac.uk
}

\begin{abstract}
A key problem in the development of information systems is understanding features of the development process. To this end, in recent years, considerable interest has been focused on modelling processes. In this paper, the results of an empirical investigation into the use of prototyping in information systems development is described. Nine prototyping processes across eight different sites of varying size were analysed and data relating to each process collected. The notation of Role Activity Diagrams (RADs) was used to capture each of the nine processes. Analysis of the interactions in each process revealed that the project manager interacted with the prototyper far more often in large developments than in small or medium-sized developments. However, significantly more interactions between the project manager and end-user were found in small-sized developments than for any other sized site. The study demonstrates how measures of business models can aid analysis of the process rather than the product and highlights the need for more empirical investigation into this and other facets of the development process. A number of lessons have been learnt from our analysis; these we also explain.
\end{abstract}

\section{Introduction}

Through participation with the end user, prototyping claims to reduce the risk of building the wrong product. Through greater understanding of requirements by the prototyper, it also claims to enhance the quality of the software product.

In this paper, a case study of the prototyping process at eight sites of varying sizes and application domains is described. The modelling notation of Role Activity Diagrams (RADs) [7] was used to model the business processes investigated and data then collected from nine processes containing a prototyping role. A high degree of autonomy and interaction with the end-user was expected of the prototyping role. The underlying motivation for the work contained in this paper stems from a number of sources. Firstly, very little research has been undertaken into the features of the prototyping process; very little is understood about the prototyping process. Secondly, only a small body of quantitative analysis has 